\title{
Monodispersive Linear Supermolecules Stabilizing Unusual Fluid Layered Phases
}

\author{
Channabasaveshwar. V. Yelamaggad*, Ammathnadu S. Achalkumar, Doddamane S. \\ Shankar Rao and S. Krishna Prasad \\ Centre for Liquid Crystal Research, Jalahalli, Bangalore 560 103, India
}

\section{Supporting information}

\section{General}

- Chemicals were obtained from Fluka, Aldrich, Lancaster and local chemical companies, and were used without any purification; solvents were dried following the standard procedures.

- Chromatography was performed using either silica gel (60-120, 100-200 and 230-400 mesh) or neutral aluminiun oxide. For thin layer chromatography, aluminium sheets pre-coated with silica gel (Merck, Kieselgel60, $\mathrm{F}_{254}$ ) were employed.

- $\quad$ IR spectra were recorded on a Perkin-Elmer Spectrum 1000 FT-IR spectrometer. The spectral positions are given in wave number $\left(\mathrm{cm}^{-1}\right)$ unit.

- NMR spectra were recorded using either a Bruker DRX -500 (500 MHz) or Bruker AMX-400 (400 MHz) spectrometer or Bruker Aveance series DPX-200 (200 MHz) spectrometer. For ${ }^{1}$ H NMR spectra, the chemical shifts are reported in ppm relative to TMS as an internal standard. Coupling constants are in $\mathrm{Hz}$.

- Microanalyses were performed using either a Euro EA3000 model elemental analyzer.

- Mass spectra were determined on JEOL JMS-600H spectrometer in FAB+ mode using 3-nitrobenzyl alcohol as a liquid matrix.

- Molecular length is calculated from the energy minimized structure deduced from CS Chem 3D version 9.0 programme. 
- X-Ray diffraction studies were carried on powder samples in Lindemann capillaries with $\mathrm{CuK}_{\alpha}$ radiation using an Image Plate Detector (MAC Science, Japan) equipped with a double mirror focusing optics.

- The mesogenic compounds were investigated for their liquid crystalline behavior employing an optical polarizing microscope (Leica DMLP) equipped with a programmable hot stage (Mettler FP90) and differential scanning calorimeter (Perkin Elmer DSC7). Measurements under electric field were carried out using glass cells of 5-10 $\mu \mathrm{m}$ thick prepared from glass plates coated with ITO transparent electrodes separated by mylar spacers. Selective reflection experiments were performed using UV-3101PC (SHIMADZU) spectrophotometer.

- Above mentioned optical polarizing microscope equipped with a programmable hot stage and differential scanning calorimeter were used to determine the melting points of non-mesomorphic compounds.

- Cyclic voltammetry measurement was carried out with $\mathrm{CH}$ instrument (Texas, USA) model 619B series computer controlled potentiostat. All the experiments were performed at room temperature and the electrochemical potential of $\mathrm{Ag}$ was calibrated with respect to ferrocene/ferrocenium $\left(\mathrm{Fc} / \mathrm{Fc}^{+}\right)$couple. A conventional three-electrode cell was used consisting of $1 \mathrm{~mm}$ diameter platinum disc working electrode, silver-silver chloride reference electrode and the platinum wire as counter electrode. The reference electrode consisted of a silver wire suspended in the electrolyte solution saturated with $\mathrm{LiCl}$. 0.1 M solution of $\mathrm{Bu}_{4} \mathrm{NPF}_{6}$ is used as buffer. Scanning rate was $0.05 \mathrm{mVs}^{-1}$ 


\section{$\underline{\text { Synthetic procedures }}$}

The detailed synthetic procedures and the molecular structural characterization data have been presented for the intermediate and target compounds in the order of schemes

\section{Scheme 1}

General procedure for the synthesis of naphthyl cholesteryl alkanoates $(\mathbf{5 a}$ and $\mathbf{5 b})$ :

A flask equipped with magnetic stirrer, reflux condenser and argon inlet was charged with cholesteryl bromoalkanoates (6a and $\mathbf{6 b}, 2.7 \mathrm{mmol}, 1.0$ equiv.), anhyd. $\mathrm{K}_{2} \mathrm{CO}_{3}$ (10.9 mmol, 4.0 equiv.), 2,4-dihydroxy naphthalene (10.9 mmol, 4.0 equiv) and dry DMF (10ml). The reaction mixture was stirred at $100{ }^{\circ} \mathrm{C}$ for 24 hours. The off white solid separated upon addition of the cooled reaction mixture into ice-cold water was collected by filtration and was dissolved in a mixture of ethyl acetate and dichloromethane. The resultant solution was washed twice with brine and dried over anhyd. $\mathrm{Na}_{2} \mathrm{SO}_{4}$. Evaporation of solvent furnished a white solid, which was purified by column chromatography using silica gel (100-200 mesh). Elution, first with hexanes followed by mixture of $15 \%$ EtOAc-hexanes afforded the product as a white solid.

5a: $R_{f}=0.43$ (25\% EtOAc -hexanes); a white solid; yield: $74.0 \%$ m.p: $155^{\circ} \mathrm{C}$; IR (KBr Pellet) $v_{\max }$ in $\mathrm{cm}^{-1} 3415,2932,1710,1606,1525,1470,1392,1332,1266$, 1253, 1222, 1028 and 742; ${ }^{1} \mathrm{H}$ NMR (200 MHz, $\left.\mathrm{CDCl}_{3}\right): \delta 7.61(\mathrm{t}, 2 \mathrm{H} J=9 \mathrm{~Hz}, \mathrm{Ar})$, 7.06-7.09 (m, 4H, Ar), 5.4 (bd, 1H, $1 \times$ olefinic H), $4.82(\mathrm{~s}, 1 \mathrm{H},-\mathrm{OH}), 4.64(\mathrm{~m}, 1 \mathrm{H}, 1$ $\times \mathrm{CHOCO}), 4.1\left(\mathrm{t}, 2 \mathrm{H}, J=6.4 \mathrm{~Hz}, 1 \times \mathrm{OCH}_{2}\right), 2.35(\mathrm{t}, 4 \mathrm{H}, J=7.0 \mathrm{~Hz}, 2 \times$ allylic $\left.\mathrm{CH}_{2}\right), 1.99-0.86\left(\mathrm{~m}, 42 \mathrm{H}, 12 \times \mathrm{CH}_{2}, 6 \times \mathrm{CH}, 4 \times \mathrm{CH}_{3}\right), 0.69\left(\mathrm{~s}, 3 \mathrm{H}, 1 \times \mathrm{CH}_{3}\right) ; \mathrm{MS}$ (FAB+): $\mathrm{m} / \mathrm{z}$ for $\mathrm{C}_{42} \mathrm{H}_{60} \mathrm{O}_{4}$, Calculated: 628.4, Found: 629.5 .

5b: $R_{f}=0.44$ (25\% EtOAc -hexanes); a white solid; yield:70.0\% ; m.p: $157{ }^{\circ} \mathrm{C}$; IR (KBr Pellet) $v_{\max }$ in $\mathrm{cm}^{-1} 3378,2948,2865,1734,1686,1508,1466,1384,1223$, 1112, 1027, 948, 848 and 798; ${ }^{1} \mathrm{H}$ NMR $\left(200 \mathrm{MHz}, \mathrm{CDCl}_{3}\right): \delta 7.61(\mathrm{t}, 2 \mathrm{H} J=9 \mathrm{~Hz}$, 
Ar), 7.06-7.09 (m, 4H, Ar), $5.4(\mathrm{bd}, 1 \mathrm{H}, J=3.8 \mathrm{~Hz}, 1 \times$ olefinic $\mathrm{H}), 4.82(\mathrm{~s}, 1 \mathrm{H},-$ $\mathrm{OH}), 4.64(\mathrm{~m}, 1 \mathrm{H}, 1 \times \mathrm{CHOCO}), 4.1\left(\mathrm{t}, 2 \mathrm{H}, J=6.4 \mathrm{~Hz}, 1 \times \mathrm{OCH}_{2}\right), 2.35(\mathrm{t}, 4 \mathrm{H}, J=$ 7.0 Hz, $2 \times$ allylic $\left.\mathrm{CH}_{2}\right), 1.99-0.86\left(\mathrm{~m}, 44 \mathrm{H}, 13 \times \mathrm{CH}_{2}, 6 \times \mathrm{CH}, 4 \times \mathrm{CH}_{3}\right), 0.69(\mathrm{~s}$, $\left.3 \mathrm{H}, 1 \times \mathrm{CH}_{3}\right) ; \mathrm{MS}(\mathrm{FAB}+): \mathrm{m} / \mathrm{z}$ for $\mathrm{C}_{43} \mathrm{H}_{62} \mathrm{O}_{4}$, Calculated:642.6, Found:643.6.

Procedure for the synthesis of $\mathbf{4 a}$ and $\mathbf{4 b}$ :

A $100 \mathrm{ml}$ flask equipped with water condenser and argon inlet, was charged with acetone (HPLC grade, 50ml), anhydrous potassium carbonate $(6.8 \mathrm{mmol}, 4$ equiv.), compound $\mathbf{5 a}$ or $\mathbf{5 b}$ (1.7 mmol, 1equiv.), and dibromoalkane (10.3 mmol, 6 equiv.), and then flushed with argon for some time. After closing the neck with a septum, the reaction mixture was heated to reflux for 24 hours under nitrogen atmosphere with vigorous stirring and then filtered the reaction mixture. The filtrate was evaporated and the crude mass obtained was dissolved in $\mathrm{CHCl}_{3}$, washed with brine, and dried over anhyd. $\mathrm{Na}_{2} \mathrm{SO}_{4}$. Evaporation of solvent gave an off white solid which was purified by column chromatography using silica gel (100-200 mesh). Elution with a mixture of 5\% EtOAc-hexanes yielded the pure product.

4a: $R_{f}=0.48$ (10\% EtOAc -hexanes); a white solid; yield: $74.0 \%$; m.p: $86.5^{\circ} \mathrm{C}$, IR (KBr Pellet) $v_{\max }$ in $\mathrm{cm}^{-1} 2938,2867,1723,1603,1509,1462,1394,1235,1165$, 1115 and 756; ${ }^{1} \mathrm{H}$ NMR $\left(200 \mathrm{MHz}, \mathrm{CDCl}_{3}\right): \delta 7.61(\mathrm{~d}, 2 \mathrm{H} J=8.6 \mathrm{~Hz}, \mathrm{Ar}), 7.1(\mathrm{~d}, 4 \mathrm{H}$, $J=8.8 \mathrm{~Hz}$, Ar), 5.37 (brd, $1 \mathrm{H}, J=4.4 \mathrm{~Hz}, 1 \times$ olefinic), 4.6- $4.64(\mathrm{~m}, 1 \mathrm{H}, 1 \times$ CHOCO), $4.1\left(\mathrm{t}, 2 \mathrm{H}, J=6.2 \mathrm{~Hz}, 1 \times \mathrm{OCH}_{2}\right), 4.03\left(\mathrm{t}, 2 \mathrm{H}, J=6.6 \mathrm{~Hz}, 1 \times \mathrm{OCH}_{2}\right), 3.52$ (t, $\left.2 \mathrm{H} J=6.2 \mathrm{~Hz}, 1 \times \mathrm{CH}_{2} \mathrm{Br}\right), 2.33-0.85\left(\mathrm{~m}, 52 \mathrm{H}, 17 \times \mathrm{CH}_{2}, 6 \times \mathrm{CH}, 4 \times \mathrm{CH}_{3}\right), 0.67$ (s, $\left.3 \mathrm{H}, 1 \times \mathrm{CH}_{3}\right)$; $\mathrm{MS}\left(\mathrm{FAB}+\right.$ ): $\mathrm{m} / \mathrm{z}$ for $\mathrm{C}_{47} \mathrm{H}_{69} \mathrm{BrO}_{4}$, Calculated:777.4, Found: 778.4.

4b: $R_{f}=0.50$ (10\% EtOAc -hexanes); m.p: $156^{\circ} \mathrm{C}$ a white solid; yield : 78.0\%; IR (KBr Pellet) $v_{\max }$ in $\mathrm{cm}^{-1} 3446,2942,2867,1731,1604,1509,1463,1392,1239$, 1167, 1114 and 758; ${ }^{1} \mathrm{H}$ NMR (200 MHz, $\left.\mathrm{CDCl}_{3}\right): \delta 7.61$ (d, $\left.2 \mathrm{H} J=8.6 \mathrm{~Hz}, \mathrm{Ar}\right), 7.1$ (d, $4 \mathrm{H}, J=8.8 \mathrm{~Hz}, \mathrm{Ar}), 5.37$ (brd, $1 \mathrm{H}, J=4.4 \mathrm{~Hz}, 1 \times$ olefinic), 4.6- $4.64(\mathrm{~m}, 1 \mathrm{H}, 1 \times$ CHOCO), $4.1\left(\mathrm{t}, 2 \mathrm{H}, J=6.2 \mathrm{~Hz}, 1 \times \mathrm{OCH}_{2}\right), 4.03\left(\mathrm{t}, 2 \mathrm{H}, J=6.6 \mathrm{~Hz}, 1 \times \mathrm{OCH}_{2}\right), 3.52$ 
(t, $\left.2 \mathrm{H} \mathrm{J}=6.2 \mathrm{~Hz}, 1 \times \mathrm{CH}_{2} \mathrm{Br}\right), 2.33-0.85\left(\mathrm{~m}, 52 \mathrm{H}, 17 \times \mathrm{CH}_{2}, 6 \times \mathrm{CH}, 4 \times \mathrm{CH}_{3}\right), 0.67$ (s, 3H, $\left.1 \times \mathrm{CH}_{3}\right) ; \mathrm{MS}(\mathrm{FAB}+): \mathrm{m} / \mathrm{z}$ for $\mathrm{C}_{47} \mathrm{H}_{69} \mathrm{BrO}_{4}$, Calculated: 777.4. Found: 778.5.

$\underline{\text { Procedure for the synthesis of } \mathbf{3 a} \text { and } \mathbf{3 b}}$

A mixture of $\mathbf{4 a}$ or $\mathbf{4 b}$ (0.69 mmol, 1 equiv.), 4,4'-dihydroxyazobenzene( 2.7 mmol, 4 equiv.), anhyd. $\mathrm{K}_{2} \mathrm{CO}_{3}(2.7 \mathrm{mmol}, 4$ equiv. $)$ in dry acetone $(50 \mathrm{ml})$ was stirred at $60{ }^{\circ} \mathrm{C}$ for $24 \mathrm{~h}$ under argon atmosphere. The cooled reaction mixture was filtered and concentrated. The residue was dissolved in ethyl acetate, washed with water, brine and dried over anhyd. $\mathrm{Na}_{2} \mathrm{SO}_{4}$. Evaporation of the solvent gave a yellow colored solid. The solid was boiled in methanol and filtered to remove the traces of starting material to furnish a bright yellow solid.

3a: $R_{f}=0.41$ (40\% EtOAc-hexanes); an orange solid; yield: $65.0 \%$; IR (KBr Pellet) $v_{\max }$ in $\mathrm{cm}^{-1}: 3327,2942,1732,1707,1602,1504,1468,1391,1234,1164,1146$, 1113, 845 and 549; ${ }^{1} \mathrm{H}$ NMR (400 MHz, $\mathrm{CDCl}_{3}$ ): $\delta 7.84$ (q, 4H, $J=5.2 \mathrm{~Hz}, \mathrm{Ar}$ ), 7.6 (d, 2H, $J=8.6 \mathrm{~Hz}, \mathrm{Ar}), 7.07-7.12(\mathrm{~m}, 4 \mathrm{H}, \mathrm{Ar}), 7.0$ (d, 2H, J=8.8 Hz, Ar), 6.94 (d, 2H $J=9.0 \mathrm{~Hz}, \mathrm{Ar}), 5.35(\mathrm{~d}, 1 \mathrm{H}, J=4.2 \mathrm{~Hz}, 1 \times \mathrm{OH}), 5.21(\mathrm{~s}, 1 \mathrm{H}, 1 \times \mathrm{OH}), 4.61-4.64(\mathrm{~m}$, $1 \mathrm{H}, 1 \times \mathrm{CHOCO}), 4.1\left(\mathrm{~m}, 6 \mathrm{H}, 3 \times \mathrm{OCH}_{2}\right), 1.25-2.39\left(\mathrm{~m}, 52 \mathrm{H}, 17 \times \mathrm{CH}_{2}, 4 \times \mathrm{CH}_{3}, 6 \times\right.$ $\mathrm{CH}), 0.67\left(\mathrm{~s}, 3 \mathrm{H}, 1 \times \mathrm{CH}_{3}\right)$; MS $(\mathrm{FAB}+): \mathrm{m} / \mathrm{z}$ for $\mathrm{C}_{59} \mathrm{H}_{78} \mathrm{~N}_{2} \mathrm{O}_{6}$, Calculated: 910.586 . Found: 911.54.

3b: $R_{f}=0.42$ (40\% EtOAc-hexanes); an orange solid; yield: 65.0\%; IR (KBr Pellet) $v_{\max }$ in $\mathrm{cm}^{-1}: 3327,2355,1696,1586,1505,1473,1413,1383,1254,1153,837,547$ and 467; ${ }^{1} \mathrm{H}$ NMR $\left(400 \mathrm{MHz}, \mathrm{CDCl}_{3}\right): \delta 7.85$ (q, 4H, $\left.J=5.6 \mathrm{~Hz}, \mathrm{Ar}\right), 7.61(\mathrm{~d}, 2 \mathrm{H}, J=$ 8.4 Hz, Ar), 7.08-7.12 (m, 4H, Ar), 7.0 (d, 2H, J=8.8 Hz, Ar), 6.94 (d, 2H J=8.6 Hz, Ar), $5.37(\mathrm{~d}, 1 \mathrm{H}, J=4.2 \mathrm{~Hz}, 1 \times \mathrm{OH}), 5.2(\mathrm{~s}, 1 \mathrm{H}, 1 \times \mathrm{OH}), 4.61-4.64(\mathrm{~m}, 1 \mathrm{H}, 1 \times$ $\mathrm{CHOCO}), 4.06\left(\mathrm{~m}, 4 \mathrm{H}, 2 \times \mathrm{OCH}_{2}\right), 4.05\left(\mathrm{t}, 2 \mathrm{H}, 1 \times \mathrm{OCH}_{2}\right), 1.25-2.35(\mathrm{~m}, 52 \mathrm{H}, 17 \times$ $\left.\mathrm{CH}_{2}, 4 \times \mathrm{CH}_{3}, 6 \times \mathrm{CH}\right), 0.67\left(\mathrm{~s}, 3 \mathrm{H}, 1 \times \mathrm{CH}_{3}\right) ; \mathrm{MS}(\mathrm{FAB}+): \mathrm{m} / \mathrm{z}$ for $\mathrm{C}_{59} \mathrm{H}_{78} \mathrm{~N}_{2} \mathrm{O}_{6}$, Calculated:910.6, Found: 911.6. 


\section{Scheme 2}

Procedure for the synthesis of $9 \mathbf{a}$ and $9 \mathbf{b}$ :

A $100 \mathrm{ml}$ flask equipped with water condenser and argon inlet, was charged with acetone (HPLC grade, 50ml), anhydrous potassium carbonate $(10 \mathrm{mmol}, 4$ equiv.), compound 10 ( $2.7 \mathrm{mmol}$, lequiv.), and dibromoalkane (16 mmol, 6 equiv.), and then flushed with argon for some time. After closing the neck with a septum, the reaction mixture was heated to reflux for 24 hours under nitrogen atmosphere with vigorous stirring and filtered hot through a celite bed. The filtrate was evaporated and the crude mass obtained was dissolved in $\mathrm{CH}_{2} \mathrm{Cl}_{2}$, washed with brine, and dried over $\mathrm{Na}_{2} \mathrm{SO}_{4}$. Evaporation of solvent gave an off white solid which was purified by column chromatography using silica gel (100-200 mesh). Elution with a mixture of 5\% EtOAc-hexanes yielded the pure product.

9a: $R_{f}=0.4\left(30 \% \mathrm{CH}_{2} \mathrm{Cl}_{2}\right.$-hexanes); a white solid; yield: 78.0\%; IR (KBr Pellet) $v_{\max }$ in $\mathrm{cm}^{-1}: 2923,2853,1606,1511,1467,1284,1246,1093,830,797,722$ and $534 ;{ }^{1} \mathrm{H}$ NMR (400 MHz, $\left.\mathrm{CDCl}_{3}\right): \delta 7.43(\mathrm{~d}, J=8.4 \mathrm{~Hz}, 2 \mathrm{H}, \mathrm{Ar}), 7.13(\mathrm{~d}, J=8.6 \mathrm{~Hz}, 1 \mathrm{H}, \mathrm{Ar})$, $6.84(\mathrm{~d}, J=8.6 \mathrm{~Hz}, 2 \mathrm{H}, \mathrm{Ar}), 6.6(\mathrm{~d}, J=8.4 \mathrm{~Hz}, 1 \mathrm{H}, \mathrm{Ar}), 4.15(\mathrm{t}, 2 \mathrm{H}, J=6.4 \mathrm{~Hz} 1 \times$ $\mathrm{OCH}_{2}$ ), $3.97\left(\mathrm{t}, 2 \mathrm{H} J=6.2 \mathrm{~Hz}, 3 \times \mathrm{OCH}_{2}\right), 3.43\left(\mathrm{t}, 2 \mathrm{H}, J=6.6 \mathrm{~Hz}, \mathrm{CH}_{2} \mathrm{Br}\right), 1.9-1.27$ $\left(\mathrm{m}, 70 \mathrm{H}, 35 \times \mathrm{CH}_{2}\right), 0.85\left(\mathrm{t}, 9 \mathrm{H}, J=6.6 \mathrm{~Hz}, 3 \times \mathrm{CH}_{3}\right) ; \mathrm{MS}(\mathrm{FAB}+): \mathrm{m} / \mathrm{z}$ for $\mathrm{C}_{57} \mathrm{H}_{95} \mathrm{BrO}_{4}$, Calculated:923.6, Found: 924.6.

9b: $R_{f}=0.4$ (30\% $\mathrm{CH}_{2} \mathrm{Cl}_{2}$-hexanes); a white solid; yield: 76.0\%; IR ( $\mathrm{KBr}$ Pellet) $v_{\max }$ in $\mathrm{cm}^{-1}: 2919,2849,1606,1512,1467,1376,1287,1247,1090,724,648$ and $535 ;{ }^{1} \mathrm{H}$ NMR (400 MHz, $\left.\mathrm{CDCl}_{3}\right): \delta 7.43$ (d, $\left.J=8.4 \mathrm{~Hz}, 2 \mathrm{H}, \mathrm{Ar}\right), 7.13(\mathrm{~d}, J=8.6 \mathrm{~Hz}, 1 \mathrm{H}, \mathrm{Ar}$ ), $6.84(\mathrm{~d}, 2 \mathrm{H}, J=8.6 \mathrm{~Hz}, \mathrm{Ar}), 6.6(\mathrm{~d}, 1 \mathrm{H}, J=8.4 \mathrm{~Hz}, \mathrm{Ar}), 4.15(\mathrm{t}, 2 \mathrm{H}, J=6.4 \mathrm{~Hz}, 1 \times$ $\left.\mathrm{OCH}_{2}\right), 3.97\left(\mathrm{t}, 2 \mathrm{H}, J=6.2 \mathrm{~Hz}, 3 \times \mathrm{OCH}_{2}\right), 3.43\left(\mathrm{t}, 2 \mathrm{H}, J=6.6 \mathrm{~Hz}, \mathrm{CH}_{2} \mathrm{Br}\right), 1.9-1.27$ $\left(\mathrm{m}, 68 \mathrm{H}, 34 \times \mathrm{CH}_{2}\right), 0.85\left(\mathrm{t}, 9 \mathrm{H}, J=6.6 \mathrm{~Hz}, 3 \times \mathrm{CH}_{3}\right) ; \mathrm{MS}(\mathrm{FAB}+): \mathrm{m} / \mathrm{z}$ for $\mathrm{C}_{56} \mathrm{H}_{93} \mathrm{BrO}_{4}$, Calculated: 909.6, Found: 910.6. 
Procedure for the synthesis of $8 \mathbf{a}$ and $\mathbf{8 b}$ :

A mixture of (7a or 7b) (1.1 mmol, 1 equiv.), 4,4'-dihydroxybiphenyl (4.4 mmol, 4 equiv.), anhyd. $\mathrm{K}_{2} \mathrm{CO}_{3}$ (4.4 mmol, 2 equiv.) in dry $\mathrm{DMF}$ was stirred at $85{ }^{\circ} \mathrm{C}$ for $30 \mathrm{~h}$ under argon atmosphere. The cooled reaction mixture was poured into water and the aqueous layer was extracted into $\mathrm{CH}_{2} \mathrm{Cl}_{2}(20 \mathrm{ml} \times 2)$. The combined organic layer was washed with brine dried over anhyd. $\mathrm{Na}_{2} \mathrm{SO}_{4}$. Evaporation of the solvent gave an orange colored solid, which was purified by column chromatography using silica gel (100-200 mesh). Upon elution with 10\% EtOAc-hexanes product was obtained.

8a: $R_{f}=0.38$ (20\% EtOAc-hexanes); a white solid; yield: 78.0\%; IR (KBr Pellet) $v_{\max }$ in $\mathrm{cm}^{-1}: 2918,2849,1606,1512,1467,1376,1287,1090,837$ and 534; ${ }^{1} \mathrm{H}$ NMR (400 $\left.\mathrm{MHz} \mathrm{CDCl}_{3}\right): \delta$ 7.41-7.47 (m, 6H, Ar), $7.1(\mathrm{~d}, 1 \mathrm{H}, J=8.4 \mathrm{~Hz}, \mathrm{Ar}), 6.87-6.96(\mathrm{~m}, 6 \mathrm{H}$, Ar), $6.6(\mathrm{~d}, 1 \mathrm{H}, J=8.6 \mathrm{~Hz}, \mathrm{Ar}), 4.74(\mathrm{~s}, 1 \mathrm{H}, 1 \times \mathrm{OH}), 4.16(\mathrm{t}, 2 \mathrm{H}, J=6.4 \mathrm{~Hz}, 1 \times$ $\left.\mathrm{OCH}_{2}\right), 3.94-4.04\left(\mathrm{~m}, 8 \mathrm{H}, 4 \times \mathrm{OCH}_{2}\right), 1.81-1.24\left(\mathrm{~m}, 70 \mathrm{H}, 35 \times \mathrm{CH}_{2,}\right), 0.88(\mathrm{t}, 9 \mathrm{H}, J$ $\left.=5.2 \mathrm{~Hz}, 3 \times \mathrm{CH}_{3}\right) ; \mathrm{MS}(\mathrm{FAB}+): \mathrm{m} / \mathrm{z}$ for $\mathrm{C}_{69} \mathrm{H}_{104} \mathrm{O}_{6}$, Calculated:1028.8, Found: 1029.6.

8b: $R_{f}=0.39$ (20\% EtOAc-hexanes); a white solid; yield: 79.0\%; IR (KBr Pellet) $v_{\max }$ in $\mathrm{cm}^{-1}: 3378,2919,2849,1607,1501,1466,1381,1242,1085,820$ and $509 ;{ }^{1} \mathrm{H}$ NMR (400 MHz, $\left.\mathrm{CDCl}_{3}\right): \delta$ 7.41-7.47 (m, 6H, Ar), 7.1 (d, 1H, J=8.4 Hz, Ar), 6.87$6.96(\mathrm{~m}, 6 \mathrm{H}, \mathrm{Ar}), 6.6(\mathrm{~d}, 1 \mathrm{H}, J=8.6 \mathrm{~Hz}, \mathrm{Ar}), 4.74(\mathrm{~s}, 1 \mathrm{H}, 1 \times \mathrm{OH}), 4.16(\mathrm{t}, 2 \mathrm{H}, J=$ $\left.6.4 \mathrm{~Hz}, 1 \times \mathrm{OCH}_{2}\right), 3.94-4.04\left(\mathrm{~m}, 8 \mathrm{H} 4 \times \mathrm{OCH}_{2}\right), 1.81-1.24\left(\mathrm{~m}, 68 \mathrm{H}, 34 \times \mathrm{CH}_{2}\right)$, 0.88 (t, $\left.9 \mathrm{H}, J=5.2 \mathrm{~Hz}, 3 \times \mathrm{CH}_{3}\right) ; \mathrm{MS}(\mathrm{FAB}+)$ : $\mathrm{m} / \mathrm{z}$ for $\mathrm{C}_{68} \mathrm{H}_{102} \mathrm{O}_{6}$, Calculated: 1014.8, Found:1015.6.

Procedure for the synthesis of $7 \mathbf{a}$ and $7 \mathbf{b}$ :

A 100ml flask equipped with water condenser and argon inlet, was charged with acetone (HPLC grade, $50 \mathrm{ml})$, anhydrous potassium carbonate $(2.36 \mathrm{mmol}, 4$ 
equiv.), compound $8 \mathbf{a}$ or $8 \mathbf{b}$ (0.59 mmol, lequiv.), and dibromoalkane (3.55 mmol, 6 equiv.), and then flushed with argon for some time. After closing the neck with a septum, the reaction mixture was heated to reflux for 24 hours under nitrogen atmosphere with vigorous stirring and filtered hot through a celite bed. The filtrate was evaporated and the crude mass obtained was dissolved in $\mathrm{CHCl}_{3}$, washed with brine, and dried over anhyd. $\mathrm{Na}_{2} \mathrm{SO}_{4}$. Evaporation of solvent gave an off white solid which was purified by column chromatography using silica gel (100-200 mesh). Elution with a mixture of 5\% EtOAc-hexanes yielded the pure product.

7a: $R_{f}=0.5$ (10\% EtOAc-hexanes), a white solid; yield: $80 \%$; IR (KBr Pellet) $v_{\max }$ in $\mathrm{cm}^{-1}: 2920,2852,1610,1506,1470,1384,1246,1094,1040,821$ and $763 ;{ }^{1} \mathrm{H}$ NMR (400 MHz, $\mathrm{CDCl}_{3}$ ): $\delta 7.44$ (t, 6H, $\left.J=8.4 \mathrm{~Hz}, \mathrm{Ar}\right), 7.13$ (d, $\left.1 \mathrm{H}, J=8.6 \mathrm{~Hz}, \mathrm{Ar}\right), 6.94$ (d, 4H, $J=8.6 \mathrm{~Hz}, \mathrm{Ar}), 6.84$ (d, 2H, $J=8.8 \mathrm{~Hz}, \mathrm{Ar}), 6.6(\mathrm{~d}, 1 \mathrm{H}, J=8.6 \mathrm{~Hz}, \mathrm{Ar}), 4.14$ $\left(\mathrm{t}, 2 \mathrm{H}, J=6.4 \mathrm{~Hz}, 1 \times \mathrm{OCH}_{2}\right), 3.94-3.99\left(\mathrm{~m}, 10 \mathrm{H}, 5 \times \mathrm{OCH}_{2}\right), 3.43(\mathrm{t}, 2 \mathrm{H}, J=6.8 \mathrm{~Hz}$, $\left.1 \times \mathrm{CH}_{2} \mathrm{Br}\right), 1.26-1.82\left(\mathrm{~m}, 78 \mathrm{H}, 39 \times \mathrm{CH}_{2}\right), 0.86\left(\mathrm{t}, 9 \mathrm{H}, J=5.2 \mathrm{~Hz}, 3 \times \mathrm{CH}_{3}\right) ; \mathrm{MS}$ $(\mathrm{FAB}+): \mathrm{m} / \mathrm{z}$ for $\mathrm{C}_{75} \mathrm{H}_{115} \mathrm{BrO}_{6}$, Calculated:1191.787. Found: 1192.6.

7b: $R_{f}=0.5$ (10\% EtOAc-hexanes); a pale yellow solid; yield: 78.0\%; IR (KBr Pellet) $v_{\max }$ in $\mathrm{cm}^{-1}: 2919,2851,1608,1505,1469,1383,1245,1092,1039,820$ and $760 ;{ }^{1} \mathrm{H}$ NMR (400 MHz, $\left.\mathrm{CDCl}_{3}\right): \delta 7.44(\mathrm{t}, 6 \mathrm{H}, J=8.4 \mathrm{~Hz}, \mathrm{Ar}), 7.13(\mathrm{~d}, 1 \mathrm{H}, J=8.6 \mathrm{~Hz}$, Ar), $6.94(\mathrm{~d}, 4 \mathrm{H}, J=8.6 \mathrm{~Hz}, \mathrm{Ar}), 6.84(\mathrm{~d}, 2 \mathrm{H}, J=8.8 \mathrm{~Hz}, \mathrm{Ar}), 6.6$ (d, 1H, $J=8.6 \mathrm{~Hz}$, Ar), $4.14\left(\mathrm{t}, 2 \mathrm{H}, J=6.4 \mathrm{~Hz}, 1 \times \mathrm{OCH}_{2}\right), 3.94-3.99\left(\mathrm{~m}, 10 \mathrm{H}, 5 \times \mathrm{OCH}_{2}\right), 3.43(\mathrm{t}, 2 \mathrm{H}$, $\left.J=6.8 \mathrm{~Hz}, 1 \times \mathrm{CH}_{2} \mathrm{Br}\right), 1.26-1.82\left(\mathrm{~m}, 74 \mathrm{H}, 37 \times \mathrm{CH}_{2}\right), 0.86(\mathrm{t}, 9 \mathrm{H}, J=5.2 \mathrm{~Hz}, 3 \times$ $\mathrm{CH}_{3}$ ); $\mathrm{MS}(\mathrm{FAB}+): \mathrm{m} / \mathrm{z}$ for $\mathrm{C}_{73} \mathrm{H}_{111} \mathrm{BrO}_{6}$, Calculated:1163.9, Found:1164.6.

General procedure for the preparation of pentamers $(\mathbf{1 a}, \mathbf{1 b}, \mathbf{2} \mathbf{a}$ and $\mathbf{2 b})$ :

Intermediates $\mathbf{7 a}$ or $\mathbf{7 b}(0.16 \mathrm{mmol}, 1$ equiv. $)$ on treating with $\mathbf{3 a}$ or $\mathbf{3 b}(0.16$ mmol, 1 equiv) were taken in dry DMF $(5 \mathrm{ml})$. Added anhyd. $\mathrm{K}_{2} \mathrm{CO}_{3}(0.66 \mathrm{mmol}, 5$ equiv) and the mixture was heated at $85{ }^{\circ} \mathrm{C}$ for $12 \mathrm{~h}$ under nitrogen atmosphere. 
Reaction mixture was initially clear and later turned turbid. The reaction mixture was filtered in hot condition and washed with hot DMF. Then this solid was dissolved in chloroform and given thorough water wash $(4 \times 50 \mathrm{ml})$. Later the organic layer was washed with brine and dried over $\mathrm{Na}_{2} \mathrm{SO}_{4}$. Evaporation of the solvent gave a yellow solid, which was repeatedly recrystallised in hot DMF. Similarly pentamers $\mathbf{2 a}$ and $\mathbf{2 b}$ were prepared by treating two equivalents of intermediates $7 \mathbf{a}$ or $7 \mathbf{b}(0.24 \mathrm{mmol})$, 4,4'-dihydroxyazobenzene ( $0.12 \mathrm{mmol}, 1$ equiv.) in presence of anhyd. $\mathrm{K}_{2} \mathrm{CO}_{3}(0.93$ mmol, 8 equiv) and dry DMF $(5 \mathrm{ml})$. The yellow colored solids obtained were purified by repeated recrystallization with hot DMF.

1a: $R_{f}=0.56\left(100 \% \mathrm{CHCl}_{3}\right)$; a yellow solid; yield: $49.0 \%$; UV - Vis: $\lambda \max =356.0$ $\mathrm{nm}, \varepsilon=1.833 \times 10^{4} \mathrm{~L} \mathrm{~mol}^{-1} \mathrm{~cm}^{-1}$; IR (KBr pellet): $v_{\max }$ in $\mathrm{cm}^{-1} 3632,2926,2852,2370$, 1733, 1602, 1504,1468, 1241, 1030, 844 and 728; ${ }^{1} \mathrm{H}$ NMR (400 MHz, $\left.\mathrm{CDCl}_{3}\right): \delta$ $7.87(\mathrm{~d}, 4 \mathrm{H}, J=8.9 \mathrm{~Hz}, \mathrm{Ar}), 7.62$ (d, 2H, $J=8.6 \mathrm{~Hz}, \mathrm{Ar}), 7.46$ (d, 4H, $J=8.4 \mathrm{~Hz}, \mathrm{Ar})$, $7.43(\mathrm{~d}, 2 \mathrm{H}, J=8.6 \mathrm{~Hz}, \mathrm{Ar}), 7.09-7.15$ (m, 5H, Ar), 7.02 (dd, 4H, J=2 Hz, 8.2 Hz, Ar), $6.94(\mathrm{~d}, 4 \mathrm{H}, J=2 \mathrm{~Hz}, 8.8 \mathrm{~Hz}, \mathrm{Ar}), 6.85$ (d, 2H, $J=8.5 \mathrm{~Hz}, \mathrm{Ar}), 6.61$ (d, 1H, $J=$ $8.0 \mathrm{~Hz}, \mathrm{Ar}), 5.35$ (d, 1H, J = 3.2 Hz, $1 \times$ olefinic $\mathrm{H}), 4.62(\mathrm{~m}, 1 \mathrm{H}, 1 \times \mathrm{CHOCO}), 4.12$ $\left(\mathrm{t}, 6 \mathrm{H}, J=6.4 \mathrm{~Hz}, 3 \times \mathrm{OCH}_{2}\right) 4.07\left(\mathrm{t}, 8 \mathrm{H}, J=5.8 \mathrm{~Hz}, 4 \times \mathrm{OCH}_{2}\right), 4.01(\mathrm{t}, 4 \mathrm{H}, J=7.7$ $\left.\mathrm{Hz} 2 \times \mathrm{OCH}_{2}\right), 3.97\left(\mathrm{t}, 2 \mathrm{H}, J=6.5 \mathrm{~Hz}, 1 \times \mathrm{OCH}_{2}\right), 2.4-0.67\left(\mathrm{~m}, 136 \mathrm{H}, 6 \times \mathrm{CH}_{3}, 56 \times\right.$ $\left.\mathrm{CH}_{2}, 6 \times \mathrm{CH}\right), 1.01\left(\mathrm{~s}, 3 \mathrm{H}, 1 \times \mathrm{CH}_{3}\right), 0.67\left(\mathrm{~s}, 3 \mathrm{H}, 1 \times \mathrm{CH}_{3}\right) ;{ }^{13} \mathrm{C} \mathrm{NMR}(100 \mathrm{MHz}$ $\left.\mathrm{CDCl}_{3}\right): \delta 172.71,161.12,158.96,158.27,155.49,154.12,153.99,147.18,142.17$, $139.74,133.48,132.72,129.85,128.03,127.63,127.39,124.26,122.53,119.13$, 116.07, 114.87, 114.74, 114.54, 111.17, 108.53, 107.324, 91.63, 84.73, 74.15, 73.89, 73.73, 69.0, 68.17, 68.04, 67.92, 67.58, 56.76, 56.27, 50.15, 42.37, 39.80, 39.52, $38.18,37.02,36.62,36.22$, 35.74, 34.32, 31.87, 30.49, 30.31, 29.60, 29.26, 29.16, 29.05, 28.72, 28.14, 27.95, 27.85, 26.19, 26.07, 25.96, 25.84, 24.25, 23.84, 22.79, 22.59, 22.46, 21.80, 21.05, 19.23, 18.7, 13.94, 11.82 and 5.36; Elemental analysis: calculated for $\mathrm{C}_{134} \mathrm{H}_{192} \mathrm{O}_{12} \mathrm{~N}_{2}$, (found)\%: C 79.56 (79.79), H 9.57 (9.81) and $\mathrm{N} 1.39$ (1.39)

1b: $R_{f}=0.55\left(100 \% \mathrm{CHCl}_{3}\right)$; a yellow solid; yield: $50.0 \%$; $\mathrm{UV}-\mathrm{Vis}$ : $\lambda \max =355.5$ $\mathrm{nm}, \varepsilon=2.073 \times 10^{4} \mathrm{~L} \mathrm{~mol}^{-1} \mathrm{~cm}^{-1}$; IR (KBr pellet): $v_{\max }$ in $\mathrm{cm}^{-1} 3632,2925,2853,2361$, 
1733, 1604, 1580, 1510,1469, 1243, 1033, 845 and 773; 1H NMR (400 MHz, $\left.\mathrm{CDCl}_{3}\right): \delta 7.86(\mathrm{~d}, 4 \mathrm{H}, J=8.6 \mathrm{~Hz}, \mathrm{Ar}), 7.62(\mathrm{~d}, 2 \mathrm{H}, J=8.6 \mathrm{~Hz}, \mathrm{Ar}), 7.46(\mathrm{~d}, 4 \mathrm{H}, J=$ $8.4 \mathrm{~Hz}, \mathrm{Ar}), 7.43$ (d, 2H, J = 8.6 Hz, Ar), 7.09- 7.15 (m, 5H, Ar), 6.99 (d, 4H, J = 8.2 $\mathrm{Hz}, \mathrm{Ar}), 6.94$ (d, 4H, $J=7.2 \mathrm{~Hz}, \mathrm{Ar}), 6.85$ (d, 2H, $J=8.5 \mathrm{~Hz}, \mathrm{Ar}), 6.61$ (d, 1H, $J=8.0$ $\mathrm{Hz}, \operatorname{Ar}), 5.35(\mathrm{~d}, 1 \mathrm{H}, J=3.2 \mathrm{~Hz}, 1 \times$ olefinic H), $4.62(\mathrm{~m}, 1 \mathrm{H}, 1 \times \mathrm{CHOCO}), 4.07(\mathrm{t}$, $\left.8 \mathrm{H}, J=5.8 \mathrm{~Hz}, 4 \times \mathrm{OCH}_{2}\right), 4.01\left(\mathrm{t}, 4 \mathrm{H}, J=6.5 \mathrm{~Hz}, 2 \times \mathrm{OCH}_{2}\right), 2.4-0.67(\mathrm{~m}, 132 \mathrm{H}, 6$ $\left.\times \mathrm{CH}_{3}, 54 \times \mathrm{CH}_{2}, 6 \times \mathrm{CH}\right), 1.01\left(\mathrm{~s}, 3 \mathrm{H}, 1 \times \mathrm{CH}_{3}\right), 0.67\left(\mathrm{~s}, 3 \mathrm{H}, 1 \times \mathrm{CH}_{3}\right) ;{ }^{13} \mathrm{C} \mathrm{NMR}$ $\left(100 \mathrm{MHz} \mathrm{CDCl}_{3}\right): \delta 172.94,169.40,161.18,158.98,158.33,155.67,154.05,147.27$, $139.82,133.55,132.78,130.04,129.96,128.11,127.70,127.45,124.34,122.58$, 119.23,119.12, 116.15,114.92, 114.80,114.59,111.08, 108.56, 107.36, 91.68, 84.79, 74.21,73.85,69.05, 68.20, 67.97, 67.67, 56.81,56.32, 50.21,42.42, 39.86, 39.58, 38.24, $37.08,36.67,36.27,35.79,34.64,31.92,30.54,30.36,29.66,29.33,28.20,28.0,27.90$, 26.14, 25.90, 25.74, 24.85,24.30, 23.89, 22.64, 22.51, 21.10,19.28,18.75, 14.0, 11.9 and 11.20; MS (FAB+): m/z for $\mathrm{C}_{132} \mathrm{H}_{188} \mathrm{O}_{12} \mathrm{~N}_{2}$, Calculated: 1993.4, Found:1995.9; Elemental analysis: calculated (found)\%: C 79.47 (79.28), H 9.49 (9.19) and N 1.40 (1.43)

2a: $R_{f}=0.36\left(50 \% \mathrm{CHCl}_{3}\right.$-hexanes); a yellow solid; yield: $60.0 \%$; $\mathrm{UV}-\mathrm{Vis}$ : $\lambda \max =$ $359.0 \mathrm{~nm}, \varepsilon=2.4742 \times 10^{4} \mathrm{~L} \mathrm{~mol}^{-1} \mathrm{~cm}^{-1}$; $\mathrm{IR}$ (KBr pellet): $v_{\max }$ in $\mathrm{cm}^{-1} 2925,2855,2399$, 1604, 1580,1499, 1470, 1273, 1243, 1092, 827, 759 and 669; ${ }^{1} \mathrm{H}$ NMR (400 MHz, $\left.\mathrm{CDCl}_{3}\right): \delta 7.86(\mathrm{~d}, 4 \mathrm{H}, J=8.84 \mathrm{~Hz}, \mathrm{Ar}), 7.46(\mathrm{~d}, 8 \mathrm{H}, J=8.6 \mathrm{~Hz}, \mathrm{Ar}), 7.43(\mathrm{~d}, 4 \mathrm{H}, J=$ $8.7 \mathrm{~Hz}, \mathrm{Ar}), 7.13$ (d, 2H, $J=8.7 \mathrm{~Hz}, \mathrm{Ar}), 6.99$ (d, $J=8.8 \mathrm{~Hz}, 4 \mathrm{H}, \mathrm{Ar}), 6.94$ (dd, 8H, $J$ $=2.6 \mathrm{~Hz}, 7.6 \mathrm{~Hz}, \mathrm{Ar}), 6.85$ (d, 4H, $J=8.6 \mathrm{~Hz}, \mathrm{Ar}), 6.6$ (d, 2H, $J=8.5 \mathrm{~Hz}, \mathrm{Ar}), 4.14$ (t, $\left.12 \mathrm{H}, J=6.6 \mathrm{~Hz}, 3 \times \mathrm{OCH}_{2}\right), 3.95-4.03\left(\mathrm{~m}, 16 \mathrm{H}, 8 \times \mathrm{OCH}_{2}\right), 1.23-1.87(\mathrm{~m}, 156 \mathrm{H}, 78$ $\left.\times \mathrm{CH}_{2}\right), 0.88\left(\mathrm{t}, 18 \mathrm{H}, J=6.0 \mathrm{~Hz}, 6 \times \mathrm{CH}_{3}\right) ;{ }^{13} \mathrm{C} \mathrm{NMR}\left(100 \mathrm{MHz} \mathrm{CDCl}_{3}\right): \delta$ 161.24,159.04 ,158.37, 154.12, 154.08, 147.24, 133.57, 132.81, 127.71,127.48, $124.35,116.16,114.95,114.83,114.62,111.02,108.62,91.72,84.82,74.23,73.82$, $69.09,68.30,68.13,31.95,30.57,30.39,29.69,29.35,26.16,26.05,25.93,22.67$ and 14.02; Elemental analysis: calculated for $\mathrm{C}_{162} \mathrm{H}_{238} \mathrm{O}_{14} \mathrm{~N}_{2}$, (found)\%: C 79.82 (80.02), H 9.84 (10.09) and N 1.15 (1.12)

2b: $R_{f}=0.35$ (50\% $\mathrm{CHCl}_{3}$-hexanes); a yellow solid; yield: $55.0 \%$; UV -Vis: $\lambda \max =$ $359.5 \mathrm{~nm}, \varepsilon=1.992 \times 10^{4} \mathrm{~L} \mathrm{~mol}^{-1} \mathrm{~cm}^{-1}$; IR (KBr pellet): $v_{\max }$ in $\mathrm{cm}^{-1} 3631,2921,2851$, 
$2362,1657,1608,1580,1469,1379,1325,1274,1249,1034,827,775$ and $721 ;{ }^{1} \mathrm{H}$ NMR (400 MHz, $\mathrm{CDCl}_{3}$ ): $\delta 7.87(\mathrm{~d}, 4 \mathrm{H}, J=8.72 \mathrm{~Hz}, \mathrm{Ar}), 7.46(\mathrm{~d}, 8 \mathrm{H}, J=8.24 \mathrm{~Hz}$, Ar), 7.43 (d, 4H, $J=8.76 \mathrm{~Hz}, \operatorname{Ar}), 7.13$ (d, 2H, $J=8.64 \mathrm{~Hz}, \mathrm{Ar}), 6.98$ (m, 4H, Ar), $6.94(\mathrm{dd}, 8 \mathrm{H}, J=2.9 \mathrm{~Hz}, 8.6 \mathrm{~Hz}, \operatorname{Ar}), 6.85(\mathrm{~d}, 4 \mathrm{H}, J=8.72 \mathrm{~Hz}, \mathrm{Ar}), 6.6(\mathrm{~d}, 2 \mathrm{H}, J=$ $8.72 \mathrm{~Hz}, \mathrm{Ar}), 4.15\left(\mathrm{t}, 12 \mathrm{H}, J=6.4 \mathrm{~Hz}, 6 \times \mathrm{OCH}_{2}\right) 3.96-4.0(\mathrm{t}, 16 \mathrm{H}, J=6.4 \mathrm{~Hz}, 8 \times$ $\left.\mathrm{OCH}_{2}\right), 1.24-1.93\left(\mathrm{~m}, 148 \mathrm{H}, 74 \times \mathrm{CH}_{2}\right), 0.88\left(\mathrm{t}, 18 \mathrm{H}, J=4.8 \mathrm{~Hz}, 6 \times \mathrm{CH}_{3}\right) ;{ }^{13} \mathrm{C} \mathrm{NMR}$ $\left(100{\left.\mathrm{MHz} \mathrm{CDCl}_{3}\right)}^{2} \delta \quad 161.17,158.99,158.34,154.50,154.05,147.25,142.23\right.$, $133.65,132.78,127.70,127.45,124.33,116.17,114.94,114.81,114.60,111.23$, 108.59, 91.68, 84.81, 74.21, 73.79, 69.07, 68.21, 68.02, 31.91, 30.54, 30.37,29.66, 29.33, 29.11, 26.24, 26.14,25.90, 22.79, 22.64 and 13.99; Elemental analysis: calculated for $\mathrm{C}_{158} \mathrm{H}_{230} \mathrm{O}_{14} \mathrm{~N}_{2}$, (found)\%: C 79.68 (79.99), $\mathrm{H} 9.73$ (9.80) and $\mathrm{N} 1.17$ (1.11). 


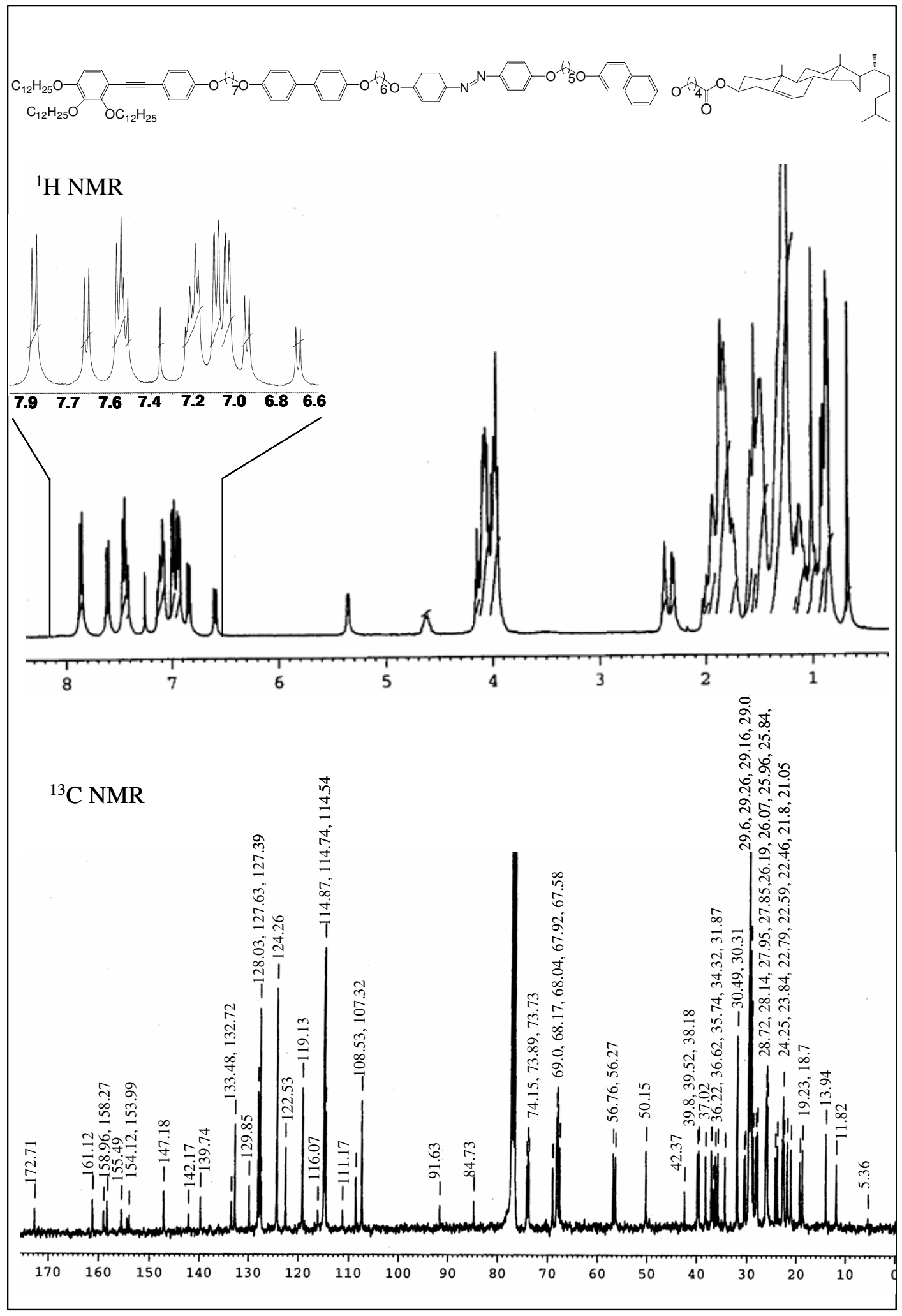

Figure 1. ${ }^{1} \mathrm{H}$ and ${ }^{13} \mathrm{C}$ NMR spectra of $\mathbf{1 a}$ 


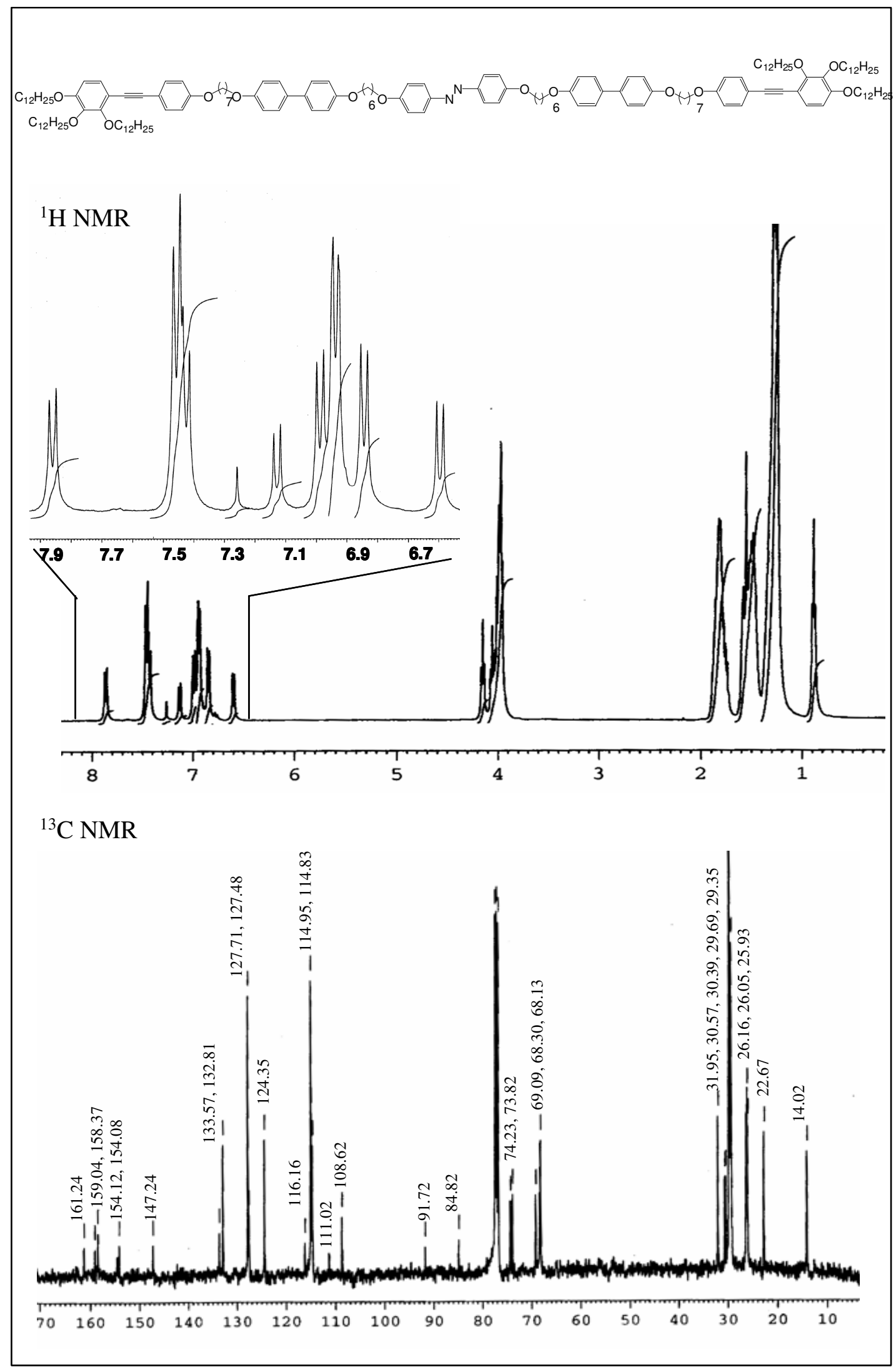

Figure 2. ${ }^{1} \mathrm{H}$ and ${ }^{13} \mathrm{C}$ NMR spectra of $\mathbf{1 b}$ 


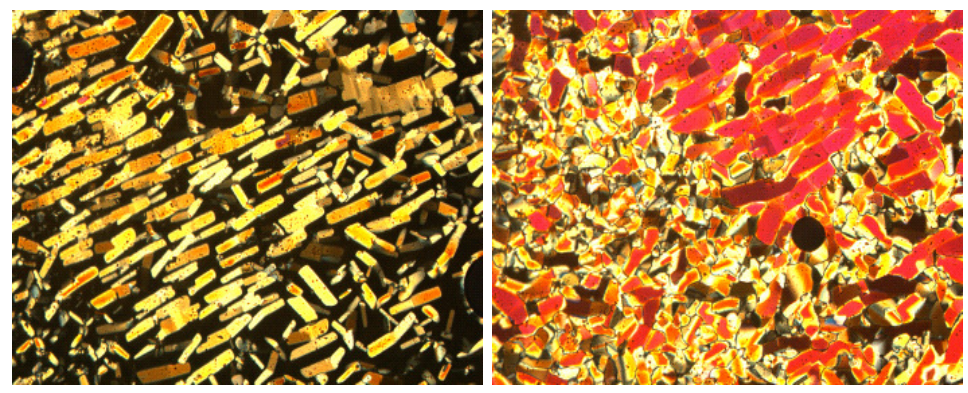

Figure 3. Photomicrographs of 2a a) Batonnets growing from isotropic state at $173{ }^{\circ} \mathrm{C}$ b) Fully developed mosaic texture at $170{ }^{\circ} \mathrm{C}$

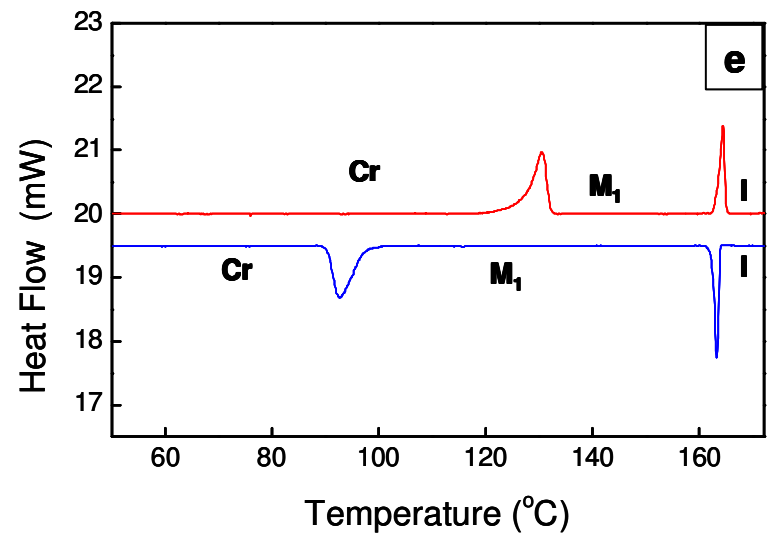

Figure 4. DSC traces obtained for the first heating and cooling cycles of the pentamer of $1 \mathrm{a}$ at a rate of $5^{\circ} \mathrm{C} \mathrm{min}{ }^{-1}\left(\mathrm{Cr}=\right.$ Crystal, $\mathrm{M}_{1}=$ Mesophase, $\mathrm{I}=$ Isotropic $)$

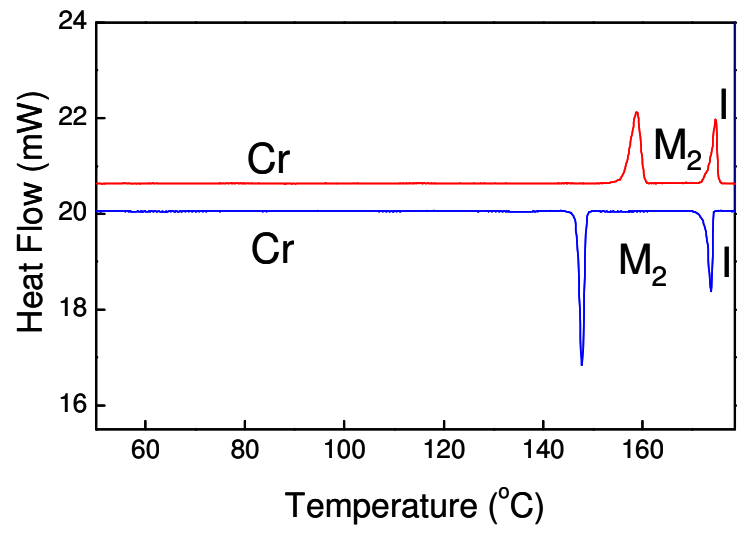

Figure 5. DSC traces obtained for the first heating and cooling cycles of the pentamer of $2 \mathbf{a}$ at a rate of $5^{\circ} \mathrm{C} \mathrm{min}{ }^{-1}\left(\mathrm{Cr}=\right.$ Crystal, $\mathrm{M}_{2}=$ Mesophase, $\mathrm{I}=$ Isotropic $)$ 

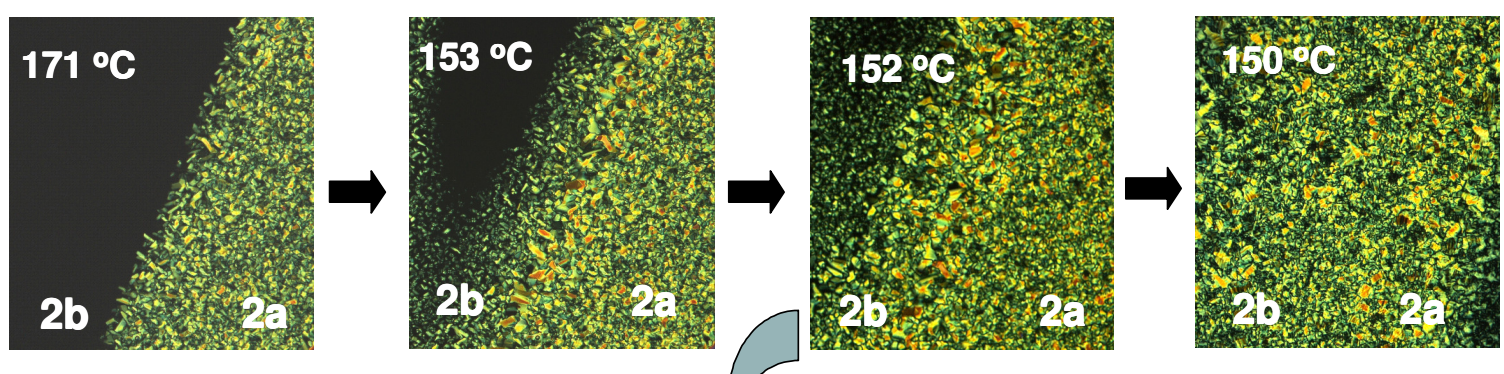

Figure 6: Microphotographs obtained during the mesophase miscibility study under POM during cooling. Notice that the mesophase formed by pentamers $2 \mathrm{a}$ and $2 \mathrm{~b}$ readily miscible.
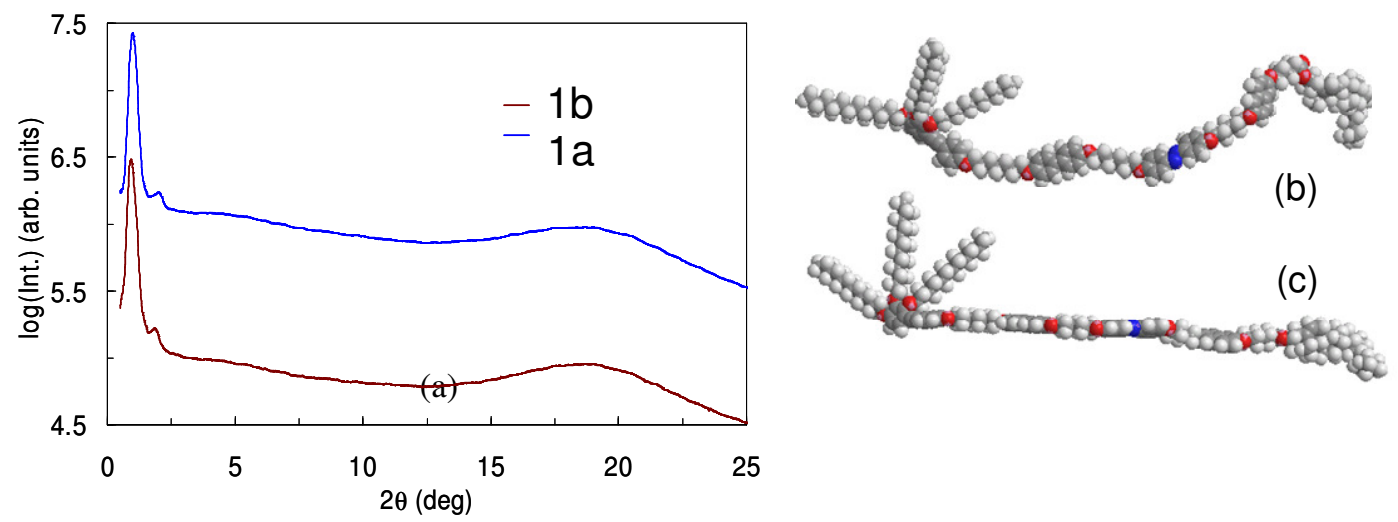

Figure 7. a) One dimensional intensity vs. $2 \theta$ profile derived from the XRD patterns obtained for the pentamers $\mathbf{1 b}\left(\right.$ at $160{ }^{\circ} \mathrm{C}$ ) and $\mathbf{1 a}\left(\right.$ at $150{ }^{\circ} \mathrm{C}$ ); Energy minimized structures of b) $\mathbf{1 a}$ and c) $\mathbf{1 b}$ 

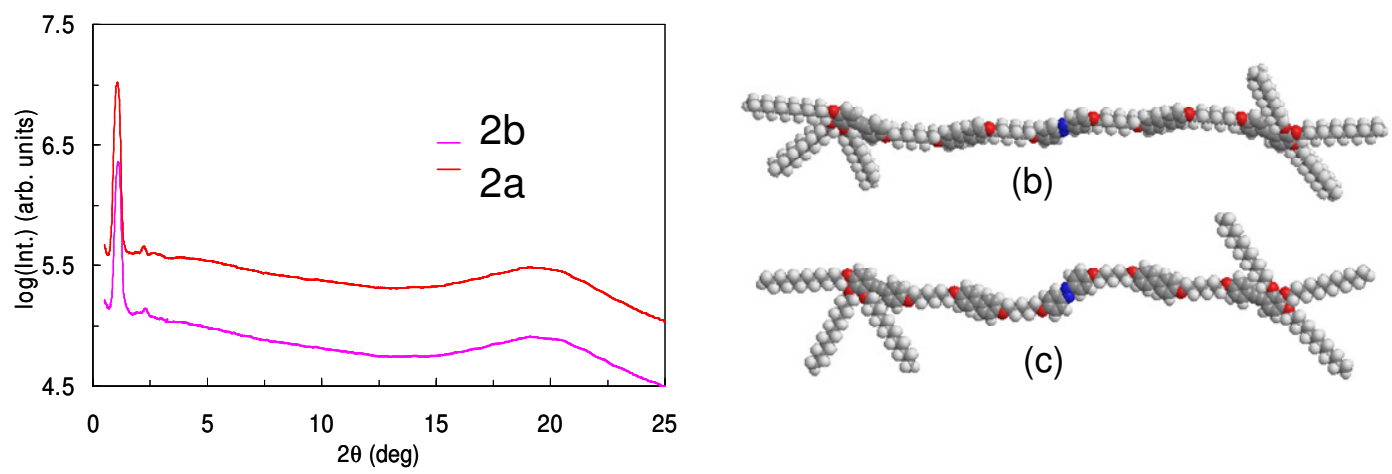

(a)

Figure 8. a) One dimensional intensity vs. $2 \theta$ profile derived from the XRD patterns obtained for the pentamers $2 \mathbf{a}$ (at $160{ }^{\circ} \mathrm{C}$ ) and $\mathbf{2 b}\left(\right.$ at $140{ }^{\circ} \mathrm{C}$ ); Energy minimized structures of b) $\mathbf{2 a}$ and c) $\mathbf{2 b}$
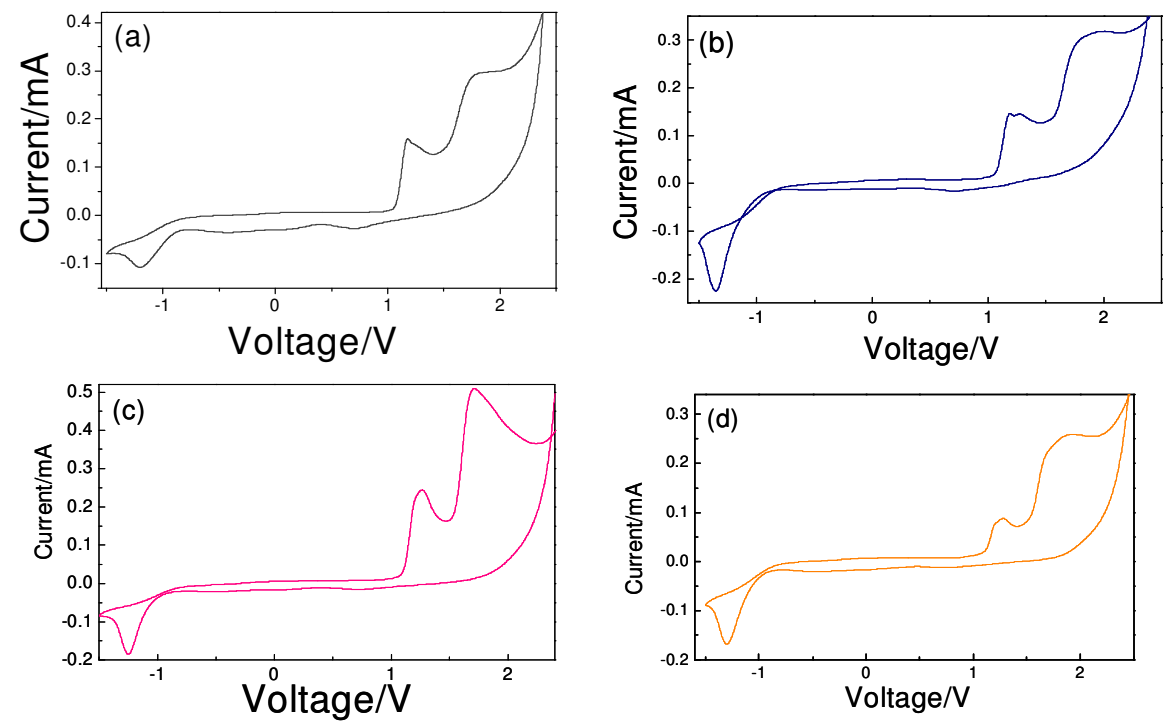

Figure 8. Cyclic Voltammograms of (a) 1a (b) 1b (c) 2a and (d) 2b; Experimental conditions: Dichloromethane, $\mathrm{Pt}$-disc working electrode, Pt-rod counter electrode, $\mathrm{Ag} / \mathrm{AgCl}$ in saturated $\mathrm{LiCl}$ solution as reference electrode, $(\mathrm{Bu})_{4} \mathrm{NPF}_{6}(0.1 \mathrm{M})$, scan rate $=100 \mathrm{mVs}-1$, room temperature. 

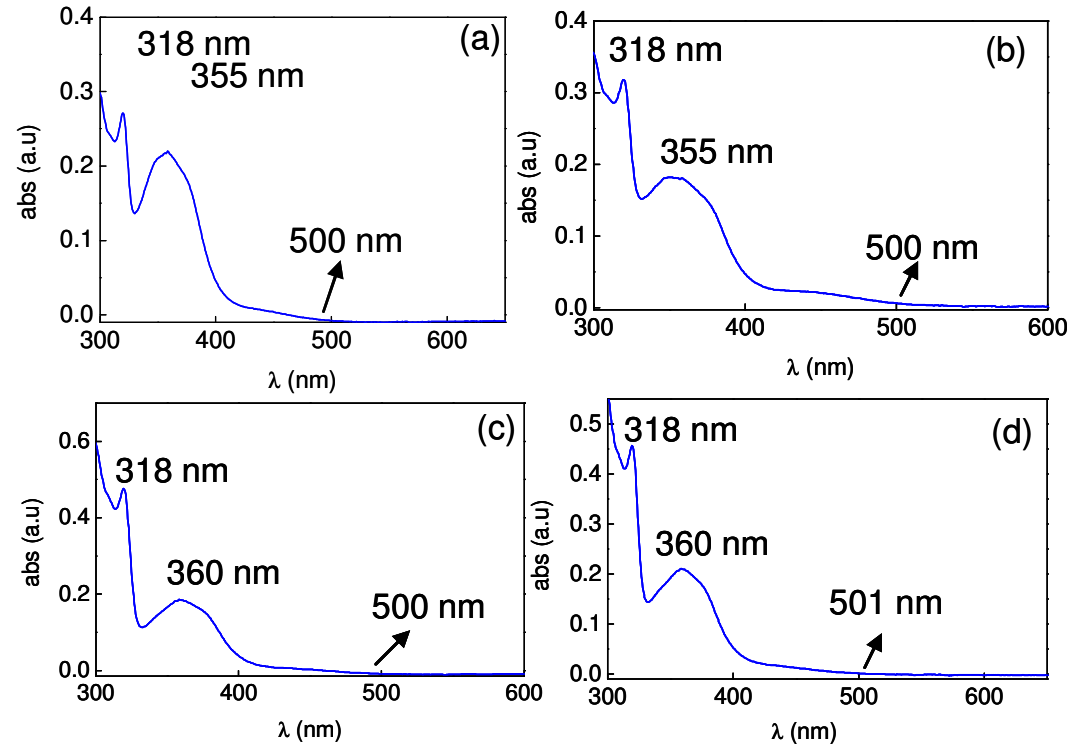

Figure 9. Absorption spectra of compounds (a) 1a; (b) 1b; (c) 1c and (d) 1d taken in micromolar solutions in dichloromethane 